\title{
Analysis of U-Shape Patterns in RR-Interval Time Series During Sleep
}

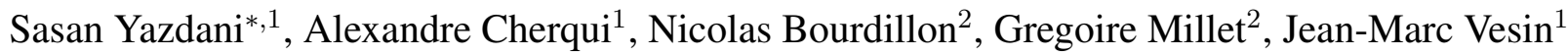 \\ ${ }^{1}$ Applied Signal Processing Group, Swiss Federal Institute of Technology, Lausanne, Switzerland \\ ${ }^{2}$ ISSUL, Institute of Sport Sciences, Faculty of Biology and Medicine, Unil, Lausanne, Switzerland
}

\begin{abstract}
The proposed study investigates a phenomenon, defined as "U-patterns", that takes place in the RR-interval time series during sleep. These patterns are defined as a $U$ shaped decrease-increase in the RR-intervals, with a duration of 20 to 40 seconds with a minimum decrease of $15 \%$ in the local RR-interval mean value. This paper studies statistical characteristics of U-patterns on subjects undergoing sleep deprivation. 15 healthy subjects (7males, $22.1 \pm 1.7$ yrs.) participated in an experiment over a span of 17 days, in three successive stages. A baseline phase of seven days, during which the subjects slept normally; A sleep deprivation phase of three days, during which they could only sleep three hours per night; Finally, in a 7day recovery phase subjects went back to sleeping normally, as they would in the baseline phase. While sleeping, polysomnographic data was recorded from the participants. U-patterns were extracted and their statistical characteristics were analyzed. Alongside the incidence of these patterns, their depth, duration and area were measured. U-patterns were present in all participating subjects. Moreover, these patterns were recurrent in all RRinterval time series. There was a significant difference in their repetition rate, depth and duration from baseline to sleep-deprivation and recovery. Results show that the characteristics of U-patterns change when subjects are undergoing sleep deprivation, suggesting these patterns can be used to identify patients suffering from sleep disorders.
\end{abstract}

\section{Introduction}

In recent years, sleep assessment has gained momentum in clinical practice. Sleep studies record human physiological parameters and through analysis, provide insightful information on possible disorders. A comprehensive sleep analysis is made possible using polysomnography (PSG), which consists in simultaneously recording several biophysiological signals during sleep. These recordings usually comprise different body function parameters such as the electrical activity of the brain, i.e. electroencephalography (EEG), by placing electrodes on the scalp [1]. Eye movement can also be recorded through electrooculography (EOG) by placing electrodes above and below each eye. Moreover, muscle activity and heart rhythm are monitored, respectively via EMG and ECG recordings.

Sleep assessment has proven useful in practice, with studies showing that sleep deprivation can lead to negative physiological effects such as myocardial hypertrophy [2], cognitive impairment [3] and hypertension [4]. Studies have demonstrated the efficacy of PSG analysis in other disorders such as sleep apnea [5,6], insomnia [7], fatigue [8].

Although numerous studies have been performed in the context of sleep analysis, research on different aspects of this important field is still much active. This is especially true for ECG-based sleep analysis, which has received less attention in comparison with EEG-based techniques. This paper investigates a phenomenon that takes place during sleep, namely U-patterns in the RR-interval time series. The state-of-the-art has not addressed this phenomenon to a meaningful degree. Very recently, Solinski et al. [9] reported the existence of these U-shaped patterns and showed they have a significant influence on the power in the very low frequency band of HRV (i.e. [0.01-0.04] $\mathrm{Hz}$ ). Mendez et al. [10] observed a quickening of heart rate during EEG A-phases, recurrent events during sleep that last between 2-60 seconds (average duration of $7 \mathrm{sec}-$ onds) [11,12], and showed there is a significant difference in HRV indexes between a group of healthy subjects and patients suffering from nocturnal front lobe epilepsy.

The remainder of the paper is organized as follows. Section 2 describes the research material used in this study. Upattern delineation and its technical aspects are explained in section 3. In Section 4, results are presented followed by a discussion in Section 5. Finally in Section 6, the main conclusions of this work are drawn.

\section{Research Material}

The data used in this study were collected from 15 subjects ( 7 males and 8 females), whose anthropometric parameters are reported in Table 1.

This study was carried out over a span of 17 days, in 
Table 1. Anthropometric parameters of this study.

\begin{tabular}{|c|c|c|}
\hline Characteristic & mean $\pm \operatorname{std}(N=15)$ & Range \\
\hline \hline Age $($ years $)$ & $22.1 \pm 1.7$ & $18-25$ \\
\hline Height $(\mathrm{cm})$ & $172.7 \pm 8.8$ & $160-196$ \\
\hline Weight $(\mathrm{kg})$ & $65.9 \pm 11.6$ & $52-92$ \\
\hline
\end{tabular}

three successive stages. First a "baseline" phase lasting for seven days, during which the subjects slept normally with no constraints. Following the baseline, subjects went through a "sleep deprivation" phase with a duration of three days, during which they could only sleep three hours at night. Finally, a 7-day "recovery" phase took place, during which subjects slept normally, as they would in the baseline phase. At the end of each phase, subjects underwent a night-long PSG recording.

\section{Methods}

\subsection{U-Patterns}

Heartbeats were first extracted from night-long ECG recordings using a non-linear R-wave detector [13], which works on a filtering technique known as relative energy (Rel-En) and has proven to provide robust results in several applications [14-16]. After the extraction of RR-interval time series from night-long ECG recordings one can observe a phenomenon, which within the context of this study is referred to as U-patterns. These patterns are recurrent within the RR-intervals and do not seem to take place periodically or quasi-periodically. This work defines these patterns as U-shape decrease-increase in the RR-interval time series, with a duration of 20 to 40 seconds, and with a minimum decrease of $15 \%$ in the local RR-interval mean value. Figure 1 illustrates an example extracted from a PSG recording. In this figure, a U-pattern with a duration of 27 seconds and a decrease in mean RR-intervals of $31 \%$, from a mean $1004 \mathrm{~ms}$ down to a mean of $693 \mathrm{~ms}$, is illustrated.

\subsection{U-Pattern Extraction}

The extracted RR-interval time series were regularly resampled at $1 \mathrm{~Hz}$, and further smoothed by applying a moving median filter with a duration of 15 seconds in order to remove small excursions. For the remainder of this paper "RR-intervals" refers to the median filtered version of this time series, unless stated otherwise. For U-pattern extraction, the RR-interval time series was used as input to the Rel-En algorithm [14] with the following empirically chosen parameters: short-term window duration of $50 \mathrm{sec}-$ onds, long-term window duration of 2000 seconds, an exponent of 10 and a Hamming window smoothing function. In order for the U-patterns to manifest as a surge in the local energy, the inverse RR-intervals were used as the input to the Rel-En algorithm. A hysteresis comparator was then applied to the output of the Rel-En algorithm. This comparator is a suitable choice as it is insensitive to small RR-intervals fluctuations around the detection threshold. Finally, the extracted events were analyzed in terms of duration and depth to delineate U-patterns. Using the output of the hysteresis comparator, U-pattern candidates were checked to have a duration of 15 to 45 seconds (a 5-second detection margin was added to the definition of U-patterns, to make the detection more robust). The decrease in RRintervals amplitude was then calculated as the difference between the average RR-interval value outside each candidate (average RR-intervals value for 60 seconds preceding and following each candidate event) and the minimum RR-interval value during the candidate U-pattern. If an RR-interval amplitude decrease larger than $15 \%$ was observed, the candidate event was selected as a U-pattern. This process is illustrated in figure 2 .

\section{Results}

Following the extraction of U-patterns, their statistical characteristics were analyzed. Alongside the incidence of these patterns, their depth, duration and area were calculated. Finally, the significance in the differences between stages, i.e. baseline, sleep deprivation, and recovery, was studied. These statistics are reported next.

Table 2 reports the subject-by-subject number of Upatterns extracted for each stage. As reported in this table, one can see that these patterns were indeed present in all participating subjects, regardless of the stage. Table 3 summarizes the inter-subject U-pattern statistics (mean \pm std) obtained for each stage. Furthermore, for each statis-
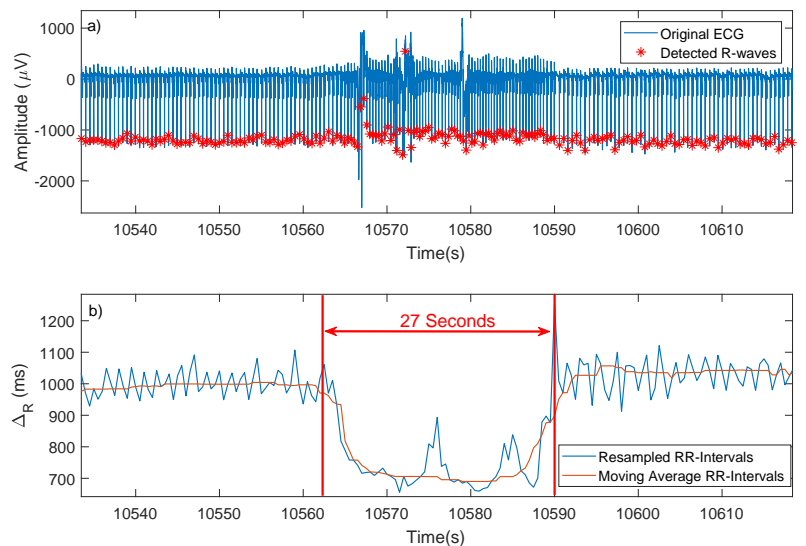

Figure 1. Illustration of a U-shaped pattern taking place during sleep. a) The ECG excerpt containing the U-pattern alongside the detected R-waves. b) The U-pattern in the RR-interval time series. 


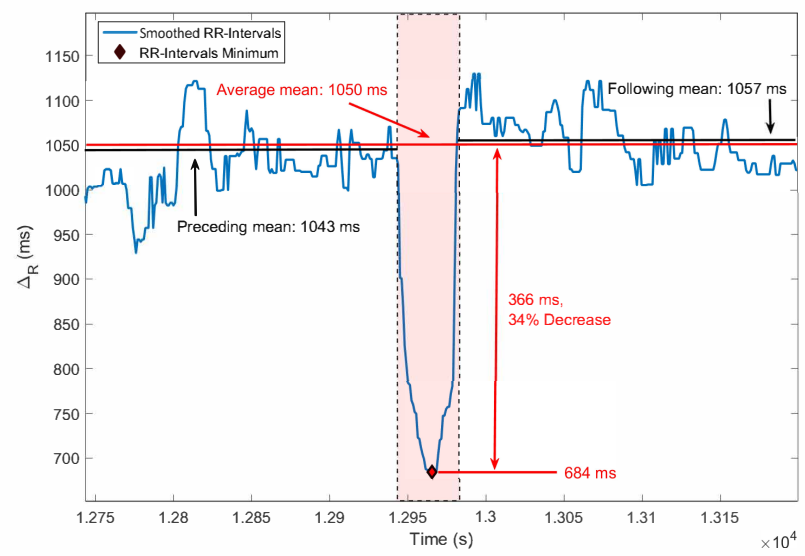

Figure 2. Extraction of U-patterns from the output of the Rel-En algorithm. The preceding and following RRinterval averages (black lines), as well as their average (red line) are depicted. In this example an amplitude decrease of $34 \%$ is observed.

tical attribute, Table 4 displays whether there was a significant inter-subject difference between different phases of the study. The hypothesis tests on sleep deprivation show significant differences across different stages. With $p$-values $<0.5$, the frequency of $\mathrm{U}$-patterns alongside their depth and integral seem to vary in a mildly meaningful way when subjects undergo different sleeping regimens.

\begin{tabular}{|c|c|c|c|c|c|c|c|c|c|c|c|c|c|c|c|}
\hline Subject & $\mathbf{1}$ & $\mathbf{2}$ & $\mathbf{3}$ & $\mathbf{4}$ & $\mathbf{5}$ & $\mathbf{6}$ & $\mathbf{7}$ & $\mathbf{8}$ & $\mathbf{9}$ & $\mathbf{1 0}$ & $\mathbf{1 1}$ & $\mathbf{1 2}$ & $\mathbf{1 3}$ & $\mathbf{1 4}$ & $\mathbf{1 5}$ \\
\hline Baseline & 31 & 42 & 39 & 38 & 20 & 36 & 48 & 35 & 17 & 23 & 21 & 36 & 49 & 33 & 43 \\
\hline Sleep deprivation & 17 & 40 & 52 & 26 & 18 & 25 & 32 & 43 & 24 & 31 & 20 & 25 & 59 & 25 & 22 \\
\hline Sleep recovery & 28 & 18 & 35 & 35 & 24 & 23 & 20 & 46 & 22 & 40 & 22 & 12 & 56 & 36 & 44 \\
\hline
\end{tabular}

Table 2. Subject-by-subject number of detected Upatterns for different sleep deprivation phases.

\begin{tabular}{|c|c|c|c|}
\hline Attribute & $\begin{array}{c}\text { Baseline } \\
(\text { mean } \pm \text { std })\end{array}$ & $\begin{array}{c}\text { Sleep Deprivation } \\
(\text { mean } \pm \text { std })\end{array}$ & $\begin{array}{c}\text { Recovery } \\
(\text { mean } \pm \text { std })\end{array}$ \\
\hline No. of U-patterns & $34.1 \pm 10.0$ & $30.6 \pm 12.5$ & $30.7 \pm 12.2$ \\
\hline U-pattern Frequency (mHz) & $1.4 \pm 0.4$ & $1.1 \pm 0.5$ & $1.3 \pm 0.4$ \\
\hline U-pattern Duration $(\mathbf{s})$ & $29.0 \pm 3.2$ & $28.4 \pm 2.4$ & $28.0 \pm 2.4$ \\
\hline U-pattern Depth $(\mathbf{m s})$ & $292.5 \pm 99.7$ & $268.9 \pm 45.1$ & $284.1 \pm 60.3$ \\
\hline U-pattern Integral (ms.s) & $4116.4 \pm 1178.5$ & $3700.8 \pm 478.0$ & $3911.8 \pm 759.6$ \\
\hline
\end{tabular}

Table 3. Inter-subject U-pattern statistical characteristics, reported for each phase of the sleep deprivation study.

\section{Discussion}

As a phenomenon taking place during sleep, U-patterns may reveal information about its quality. However, in order to draw reliable and interpretable results, the extraction of these U-shaped patterns must be addressed first. Upatterns vary in amplitude and duration, and in comparison with other complexes (such as ECG QRS-complexes), one deals with much larger temporal and amplitude variances. Therefore, linear filtering and conventional thresholding techniques may not be able to robustly detect these

\begin{tabular}{|c|c|c|c|}
\hline Attribute & $\begin{array}{c}\text { Baseline } \rightarrow \text { Sleep Deprivation } \\
p \text {-value }\end{array}$ & $\begin{array}{c}\text { Sleep Deprivation } \rightarrow \text { Recovery } \\
p \text {-value }\end{array}$ & $\begin{array}{c}\text { Baseline } \rightarrow \text { Recovery } \\
p \text {-value }\end{array}$ \\
\hline U-pattern frequency & $\mathbf{0 . 1 4}$ & $\mathbf{0 . 2 7}$ & 0.65 \\
\hline Mean U-depth & $\mathbf{0 . 1 9}$ & $\mathbf{0 . 4 4}$ & $\mathbf{0 . 3 2}$ \\
\hline Variance U-depth & $\mathbf{0 . 3 2}$ & 0.91 & $\mathbf{0 . 3 2}$ \\
\hline Mean U-duration & 0.54 & 0.66 & $\mathbf{0 . 3 3}$ \\
\hline Variance U-duration & 0.63 & 0.63 & 0.9 \\
\hline Mean U-integral & $\mathbf{0 . 1 3}$ & 0.53 & $\mathbf{0 . 2 5}$ \\
\hline Variance U-integral & $\mathbf{0 . 3 2}$ & 0.67 & $\mathbf{0 . 3 2}$ \\
\hline
\end{tabular}

Table 4. Two sample $t$-test $p$-values for each phase of the sleep deprivation study. Bold fields represent $p$-values $<0.5$.

patterns. This remains true for other physiological phenomena. The Rel-En algorithm, however, provides a robust detection technique for these patterns. When combined with a simple hysteresis comparator, this algorithm is able to robustly extract U-patterns. It uses a small number of parameters and avoids using unintuitive thresholds for U-pattern extraction.

It is worth mentioning that, although the behavior of these patterns has been cited in the literature $[9,10]$, to the best of our knowledge, this is the first study dedicated to analyze the different characteristics of U-patterns.

Statistics suggest that several U-pattern characteristics, such as frequency, depth, and duration, change significantly during sleep deprivation. Therefore, U-pattern analysis can be performed to identify subjects who are experiencing sleep disorders. Sleep quality assessment can be carried out through EEG-based sleep stage analysis. However, EEG analysis requires subjects to wear uncomfortable EEG caps connected to a recording device through several wires, causing frustration and discomfort during sleep. Analysis of U-patterns however, is only dependent upon inter-beat intervals, which can be acquired by portable PPG recording devices and from more convenient locations such as arms and fingers. More to the point, sleep deprivation is known to contribute to disease development. The biological changes induced by chronic sleep deprivation have been associated with cardiovascular and metabolic diseases, and shortened lifespan [17]. By analyzing U-patterns as another important parameter derived from portable monitoring devices, one can identify subjects who are prone, or even experiencing, sleep disorders and subsequently, prevent the development of such diseases.

\section{Conclusion}

This paper studies a U-shape acceleration/deceleration phenomenon that is discernible in the RR-intervals of night-long PSG recordings. A non-linear filtering technique is proposed for the extraction of these patterns. Statistical analysis on the characteristics of these patterns, namely their depth, duration, and the number of their incidence, is performed to analyze the effect of fatigue induced 
by sleep deprivation. Results suggest these patterns can be used to identify patients suffering from sleep disorders.

\section{Acknowledgements}

This study was performed in the framework of the NanoTera initiative supported by the Swiss National Science Foundation (SNSF).

This work was made possible by the financial support of the CTI commission $\mathrm{n}^{\circ}$ 18164.2 515 PFLS-LS.

\section{References}

[1] Niedermeyer E, da Silva FL. Electroencephalography: basic principles, clinical applications, and related fields. Lippincott Williams \& Wilkins, 2005.

[2] Fernandez-Mendoza J, Vgontzas AN, Liao D, Shaffer ML, Vela-Bueno A, Basta M, Bixler EO. Insomnia with objective short sleep duration and incident hypertension. Hypertension 2012;HYPERTENSIONAHA-112.

[3] Yaffe K, Laffan AM, Harrison SL, Redline S, Spira AP, Ensrud KE, Ancoli-Israel S, Stone KL. Sleep-disordered breathing, hypoxia, and risk of mild cognitive impairment and dementia in older women. Jama 2011;306(6):613-619.

[4] Sulit L, Storfer-Isser A, Kirchner HL, Redline S. Differences in polysomnography predictors for hypertension and impaired glucose tolerance. Sleep 2006;29(6):777-783.

[5] Fujita S, Conway W, Zorick F, Roth T. Surgical correction of anatomic abnormalities in obstructive sleep apnea syndrome: uvulopalatopharyngoplasty. Otolaryngology Head and Neck Surgery 1981;89(6):923-934.

[6] Marcus CL, Chapman D, Ward SD, McColley SA, Herrerias CT, Stillwell PC, Howenstine M, Light MJ, Schaeffer DA, Wagener JS, et al. Clinical practice guideline: diagnosis and management of childhood obstructive sleep apnea syndrome. Pediatrics 2002;109(4):704-712.

[7] Bastien CH, Vallières A, Morin CM. Validation of the insomnia severity index as an outcome measure for insomnia research. Sleep medicine 2001;2(4):297-307.

[8] Krupp LB, Jandorf L, Coyle P, Mendelson W. Sleep distur- bance in chronic fatigue syndrome. Journal of Psychosomatic Research 1993;37(4):325-331.

[9] solinski M, Gieraltowski J, Zebrowski J, Kuklik P. Influence of $u$-shape accelerations of heart rate on very low frequency band and heart rate multifractality. In 2017 Computing in Cardiology Conference (CinC). September 2017; 721-724.

[10] Dorantes-Méndez G, Mendez MO, Alba A, Parrino L, Milioli G. Time-varying analysis of the heart rate variability during a-phases of sleep: Healthy and pathologic conditions. Biomedical Signal Processing and Control 2018; 40:111-116.

[11] Terzano MG, Mancia D, Salati MR, Costani G, Decembrino A, Parrino L. The cyclic alternating pattern as a physiologic component of normal NREM sleep. Sleep September 1985; 8(2):137-145. ISSN 0161-8105.

[12] Parrino L, Ferri R, Bruni O, Terzano MG. Cyclic alternating pattern (CAP): The marker of sleep instability. Sleep Medicine Reviews February 2012;16(1):27-45. ISSN 1087-0792.

[13] Yazdani S, Vesin JM. A novel preprocessing tool to enhance ECG R-wave extraction. In The 43rd annual international conference of Computing in Cardiology. September 2016; 633-636.

[14] Yazdani S, Fallet S, Vesin J. A novel short-term event extraction algorithm for biomedical signals. IEEE Transactions on Biomedical Engineering April 2018;65(4):754762. ISSN 0018-9294.

[15] Vesin JM, Yazdani S, Mirmohamadsadeghi L, Bourdillon $\mathrm{N}$. Extraction and analysis of short-time excursions in RRinterval time series. In 2016 Computing in Cardiology Conference (CinC). September 2016; 721-724.

[16] Yazdani S, Vesin JM. An instantaneous measure of ecg signal quality. In 2018 Computing in Cardiology Conference (CinC). September 2018; 1-1.

[17] Luyster FS, Strollo PJ, Zee PC, Walsh JK. Sleep: a health imperative. Sleep 2012;35(6):727-734.

Address for correspondence:

Sasan Yazdani

EPFL SCI STI JMV - ELE 121 - Station 11

CH-1015 Lausanne - Switzerland.

E-mail address: sasan.yazdani@epfl.ch 